\title{
Secondary Switched Control with no Communications for Islanded Microgrids
}

\author{
Juan M. Rey, Pau Martí, Manel Velasco, Jaume Miret, Member, IEEE, and Miguel Castilla
}

\begin{abstract}
Flexibility and reliability of AC microgrids are enhanced by reducing the dependence on communications in multi-layer hierarchical control. In this sense, this paper presents a secondary control with no communications for islanded microgrids. It is based on a control scheme that switches between two configurations according to a time-dependent protocol. With this proposal, an excellent performance in terms of transient response and frequency restoration in steady-state is achieved. The paper also presents design considerations for the control parameters. In addition, the theoretical predictions are validated by experimental results from a laboratory microgrid.
\end{abstract}

Index Terms-Distributed generation, hierarchical control, islanded microgrids, secondary control, switched control, time-dependent protocol.

\section{INTRODUCTION}

$\mathrm{M}$ ICROGRIDS have recently emerged as a potential solution for electrical supply due to their advantages as environmental impacts reduction, reliability, and flexible operability [1], [2]. Basically, a microgrid is a cluster of distributed generators (DGs), loads, and energy storage systems which can operate in two possible modes: gridconnected or islanded [3].

In islanded mode, microgrids are disconnected from the main grid intentionally or by an electrical fault and the DGs provide active and reactive power to the local loads [4]. To control frequency, voltage amplitude or power sharing, a multi-layer hierarchical scheme can be implemented, normally

Manuscript received Month $\mathrm{xx}, 2 \mathrm{xxx}$; revised Month $\mathrm{xx}, \mathrm{xxxx}$; accepted Month $\mathrm{x}, \mathrm{xxxx}$. This work has been supported by ELAC2014/ESE0034 from the European Union and its linked Spanish national project PCIN-2015-001. We also appreciate the support from the Ministry of Economy and Competitiveness of Spain and the European Regional Development Fund (FEDER) under projects ENE2015-64087-C2-1-R and TEC2013-46938-R.

Juan M. Rey is with Escuela de Ingenierías Eléctrica, Electrónica y de Telecomunicaciones (E3T), Universidad Industrial de Santander (UIS), 680002 Bucaramanga, Colombia and with the Electronic Engineering Department, Technical University of Catalonia, Av. Victor Balaguer s/n, 08800 Vilanova i la Geltrú, Spain (e-mail: juanmrey@uis.edu.co).

Pau Marti and Manel Velasco are with the Automatic Control Department, Technical University of Catalonia, Pau Gargallo 5, 08028 Barcelona, Spain.

Jaume Miret and Miguel Castilla are with the Electronic Engineering Department, Technical University of Catalonia, Av. Victor Balaguer $s / n$, 08800 Vilanova i la Geltrú, Spain. divided in three control layers: primary, secondary and tertiary layer [5].

Primary layer rapidly controls power sharing and stabilizes frequency and output voltage amplitude in each DG. However, steady-state errors or deviations are introduced, which are corrected by the secondary layer. Tertiary layer manages and coordinates the operation of the power flows at optimal points considering economic concerns (i.e., energy prices and electricity markets) and grid requirements (i.e., reactive power flows control for voltage management) [6]-[8]. These layers decouple their dynamics through different speeds of response (i.e., control bandwidths), which also facilitates their individual designs.

In most of the literature related to multi-layer hierarchical control, primary layer approaches are based on the droop method (which is essentially decentralized). In addition, tertiary layer approaches are commonly based on centralized communication systems. On the other hand, there is no standard for secondary layer approaches, which can be categorized in centralized, distributed and with no communications.

Centralized secondary layer approach is based on a communication system which connects each DG to a microgrid central controller (MGCC) [9]. A node of the microgrid is selected as the control bus, where the frequency and voltage amplitude are measured. Its values are gathered and compared with their respective references by the MGCC. Then, secondary control terms are calculated and transmitted to the DGs controllers [10]. Commonly, the control bus is assigned at the microgrid point of common coupling which is the node where the transfer switch connects the isolated microgrid with the main grid.

As centralized approach requires an extensive communication system, it can be used for monitoring and controlling different aspects of the microgrid. This approach allows easily incorporate new DGs to the microgrid without affecting the control scheme. However, the strong dependence on the MGCC is its major drawback. As all the control calculations are done in the MGCC, a failure on it can affect the entire microgrid, leading to consider a backup system to improve the reliability.

Distributed secondary layer approach is based on a communication system which allows the exchange of data between the DGs controllers. In this approach the MGCC is not required, because the secondary control terms are calculated in each DG controller using local and received measures. Distributed approach can be implemented based on 
different control calculations techniques. Currently, two interesting approaches are averaging and consensus techniques.

In averaging technique each DG estimates its frequency, sends it to all or some other DGs, computes the average frequency with the information received and finally calculates the output of its secondary control layer [11]. A similar approach can be used to control the voltage amplitude, as is proposed in [12]. Although a MGCC is not required, averaging technique commonly implies a high traffic exchange to guarantee the control objective accomplishment.

In consensus technique, the definition of the communication interactions could be based on multi-agent systems theory, which defines the rules to govern the information exchange between an agent (DG controller) and some of its neighbors [13], [14]. Consensus technique implies a reduction in the number of communication links between DGs (only requires neighbor-to-neighbor interactions) [15]-[17], without losing robustness even in the presence of communication delays [18].

The major drawback of distributed secondary layer approach is the complexity in the physical implementation that can represent microgrids with a large number of DGs. Then, the balance between complexity and robustness of the communication system should be considered in detail during the design stage [18]-[21].

Considering the drawbacks previously presented, secondary control layer approaches with no communications emerged as a potential solution. It is important to clarify that even with a secondary control layer with no communications, a general communication system is needed for other functionalities of the microgrid as coordination of units during black start processes, real-time monitoring or tertiary control layer. However, reduction of the dependence on communications on each control layer represents an improvement on the reliability of the microgrid [22]. With less dependence on communications, the impact of the real-time data transmission over the control objectives is reduced and the security in terms of communication vulnerability risks is improved.

In [23] and [24] control schemes which perform primary, secondary, and tertiary control layers with no communications are proposed. In the former, the scheme operates with gridforming converters i.e., converters that behaves as powercontrolled voltage sources. In the latter, the scheme operates with grid-feeding converters i.e., power-controlled current sources. In [25] a secondary control with no communications is proposed and a small-signal model to study its dynamics and design considerations is developed. In these proposals, the secondary control is implemented using a low-pass filter, offering limited static and dynamic properties. In particular, fast transient response can be achieved with a high steadystate frequency deviation. Conversely, a low steady-state deviation in frequency is related with a slow transient response.

This paper proposes a secondary control with no communications for islanded microgrids. It is based on a switched control scheme and a time-dependent protocol

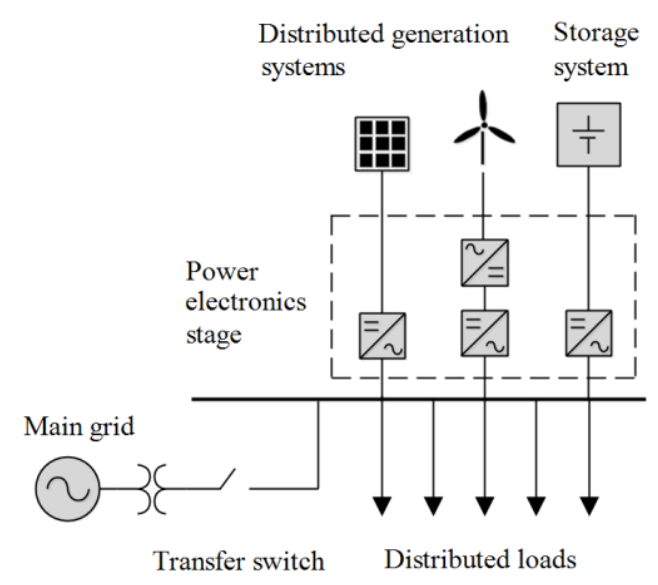

Fig. 1. General microgrid scheme

providing the outstanding features: 1) high flexibility and reliability (no communications are required to operate the secondary control), 2) fast transient response, 3) accurate frequency restoration in steady-state, and 4) simple design and implementation. As far as authors know, there are no approaches without communications providing fast transient response and accurate steady-state frequency restoration simultaneously. Experimental results from a laboratory microgrid have been reported to validate the expected features of the proposed control. This paper is organized as follows: in Section II, hierarchical control for islanded microgrids is reviewed. In Section III, the proposed control (switched control scheme with a time-dependent protocol) is presented and in Section IV its design considerations are described. Experimental results to validate the proposal are presented and discussed in Section V. Finally, general conclusions are drawn in Section VI.

\section{HIERARCHICAL CONTROL FOR ISLANDED MICROGRIDS}

Fig. 1 shows a general microgrid scheme. A microgrid works in islanded mode when it is disconnected from the main grid by the transfer switch. In this operational mode, secondary control is the highest hierarchical layer which can be implemented without communications. In this Section the control scheme for primary and secondary layers is described.

\section{A. Primary layer}

This is the first layer in the hierarchical scheme and has the fastest speed of response. The main objective of this layer is to control the power sharing and to stabilize the voltage amplitude and frequency.

The droop method is the most common control approach for primary layer, which is based on mimicking the behavior of synchronous generators, reducing the angular frequency $\omega$ by the increase of the supplied active power $P$ [26]. Also, the droop control could be implemented to relate amplitude $V$ with the supplied reactive power $Q$. The droop method is presented in the next equations, where $\omega_{0}$ is the reference angular frequency, $V_{0}$ is the reference amplitude and $m$ and $n$ are the droop control gains 


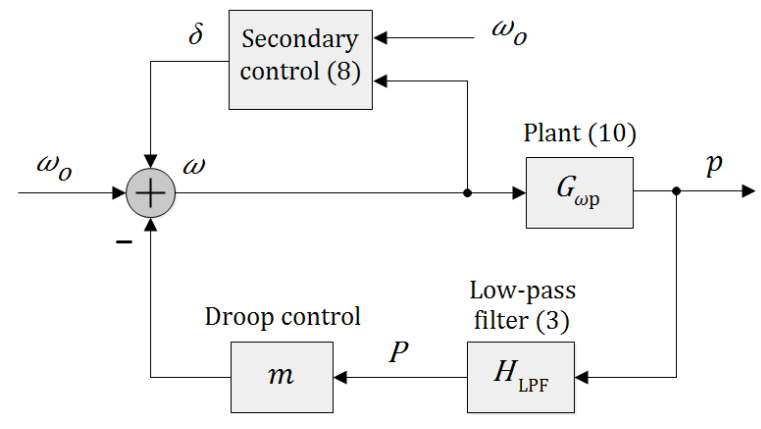

Fig. 2. Proposed control scheme for primary and secondary layers.

$$
\begin{gathered}
\omega=\omega_{0}-m P \\
V=V_{0}-n Q .
\end{gathered}
$$

To allow mismatched dynamics between the different control layers and to achieve high power quality injection, the instantaneous active power $p$ and instantaneous reactive power $q$ are filtered by the low-pass filter $H_{\mathrm{LPF}}$, as follows

$$
\begin{aligned}
& P=H_{\mathrm{LPF}} p=\frac{\omega_{c}}{s+\omega_{c}} p \\
& Q=H_{\mathrm{LPF}} q=\frac{\omega_{c}}{s+\omega_{c}} q
\end{aligned}
$$

where $\omega_{c}$ is the primary layer cut-off frequency. As the frequency is a global variable of the microgrid, the droop method ensures an accurate active power sharing in steadystate based only on local measured signals.

Reactive power sharing is controlled using a reactive power-voltage loop, which implies a difficulty to perfectly achieve the control objective using only a primary control layer. As the amplitude is not equal along the microgrid (i.e., contrary to the frequency, it is not a global variable), the profile variations affect the reactive power sharing. A uniform voltage profile through all the microgrid may cause an undesired increase in the reactive power flow. This trade-off between the voltage profile and the reactive power sharing leads to accept margins of voltage deviations based on the grid code regulation, especially in islanded microgrids where the voltage profile is not a critical control objective.

To compensate the voltage deviations and guarantee the margins of amplitude, a suitable secondary voltage control layer could be implemented based on any of the approaches presented in Section I. However, considering the limitation of the local nature of the voltage, the proposed control presented in this paper is focused only on frequency control defining as control objectives both active power sharing and frequency restoration.

\section{B. Secondary layer}

In this control layer, the steady-state errors introduced by the primary layer are compensated. To do so, an extra term is introduced in the primary layer (1) as follows

$$
\omega=\omega_{0}-m P+\delta
$$

where $\delta$ is the secondary control term.

In [23]-[25], the secondary control term is based on a lowpass filter, as follows

$$
\delta=\frac{k_{i}}{s+k_{0} k_{i}}\left(\omega_{0}-\omega\right)
$$

where $k_{i}$ and $k_{0}$ are control parameters. This transfer function is a proportional controller with an additional pole for highfrequency attenuation.

From (5) and (6), the frequency error in steady-state can be derived, as follows

$$
e_{0}=\omega_{0}-\omega=\frac{k_{0} m P}{1+k_{0}}
$$

Note that both the pole of the low-pass filter in (6) and the error $e_{0}$ in (7) depend directly of the control gain $k_{0}$. This means that this controller exhibits a design trade-off between transient response and accuracy. Next Section introduces the proposed control scheme that breaks this design trade-off providing fast transient response and small error in steadystate, simultaneously.

\section{PROPOSED CONTROL}

In this Section, the proposed secondary switched control scheme and the time-dependent protocol are presented. These control structures constitute the first (and main) contribution of this work.

\section{A. Switched control scheme}

The solution presented is based on a control that switches between two configurations, taking advantage of the features of different approaches: filtered proportional controller and integral controller. The proposed switched control can be expressed as follows

$$
\delta(t)=k_{i} \int\left[\left(\omega_{0}-\omega(t)\right) \operatorname{sgn}(k(t))-k(t) \delta(t)\right] d t
$$

where $k_{i}$ and $k(t)$ are the control parameters.

The terms of the integral function depend on the value of $k(t)$ which is controlled by the time-dependent protocol presented in the next subsection. The control system can be expressed in the Laplace domain according to the value of $k(t)$ as follows

$$
\delta=\left\{\begin{array}{cl}
\frac{k_{i}}{s+k k_{i}}\left(\omega_{0}-\omega\right) & k(t)>0 \\
C & k(t)=0
\end{array}\right.
$$

being $C$ a constant value that will be discussed latter. For positive values of $k(t)$, secondary control corresponds to a proportional low-pass filter control with a cut-off frequency determined by $k(t)$.

In Fig. 2 the proposed control scheme for primary and secondary layers is presented. The block $G_{\omega \mathrm{p}}(s)$ represents the DG plant (frequency to active power transfer function), the 


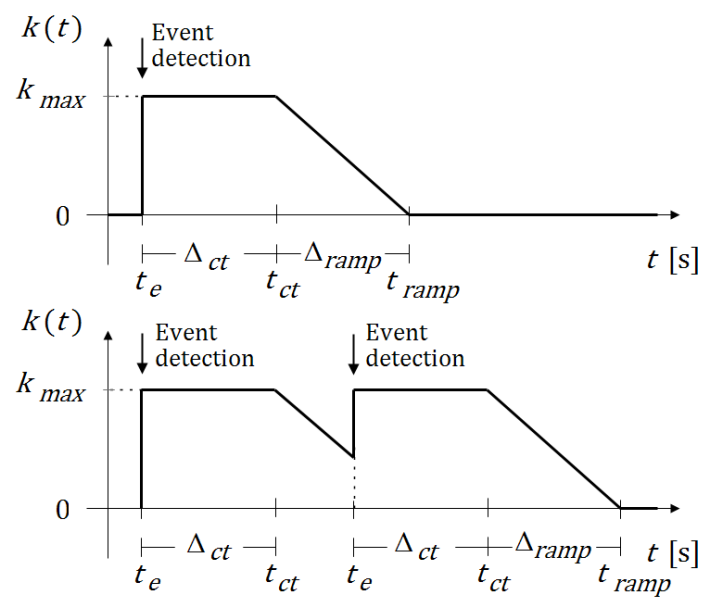

Fig. 3. Time-dependent protocol in single (top) and multi-event (bottom) scenarios.

block $H_{\mathrm{LPF}}(s)$ is the low-pass filter presented in (3) and the block $m$ corresponds to the droop control presented in (5).

For values of $k(t)$ close to 0 , the control behaves similarly to an integral controller. This type of controller allows to get a low steady-state error, however suffers from several problems (e.g., hunting phenomenon, different values for initial conditions and mismatch in control gains) that can lead to an unstable response. In fact, in this type of controllers, even low errors in the estimation of frequency $\omega$ contribute to a cumulative increase of the secondary term $\delta$. However, the switched characteristic of the proposed control avoids this drawback.

According to (8) the switching is performed by the term $\operatorname{sgn}(k(t))$. When $k(t)=0$ the input of the integral is set to 0 and the secondary layer term $\delta$ remains constant in the last value calculated, represented in (9) as $C$. Thus, hunting phenomenon is avoided. With a suitable design of the timedependent protocol (which drives the value of $k(t)$ ) it is possible to use the most convenient features of both filtered proportional controller and integral controller, achieving a fast dynamic and a negligible steady-state error.

\section{B. Proposed time-dependent protocol}

The values of the parameters $k_{i}$ and $k(t)$ affect the static and dynamic response of the secondary layer. Specifically, assuming $k_{i}$ constant, $k(t)$ determines the cut-off frequency of the low-pass filter on (9) and therefore the frequency error in steady-state. Thus, a high value of $k(t)$, which means a high bandwidth, leads to a fast dynamics but an inaccurate steady-state response. A low value of $k(t)$, which means a low bandwidth, leads to a slow dynamics but an accurate steady-state response. This design trade-off is shared by the proportional controllers reported in literature [23]-[25].

Fig. 3 shows the proposed time-dependent protocol which breaks this design trade-off, in which $k(t)$ varies temporarily once an event is detected. For a single event (top of Fig. 3), once it is detected (at $t_{e}$ ), the parameter $k(t)$ varies instantaneously from 0 to $k_{\text {max }}$. Then, $k(t)$ remains constant during $\Delta_{c t}$ seconds until $t=t_{c t}$. After that, $k(t)$ decreases linearly from $k_{\max }$ to 0 during $\Delta_{\text {ramp }}$ seconds until

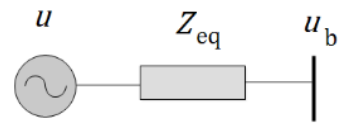

Fig. 4. Equivalent circuit of an electronically-coupled DG system

$t=t_{\text {ramp }}$. At $t_{\text {ramp }}$ the input of the integral term of (8) is set to $0(\operatorname{sgn}(0)=0)$, which means that the secondary term $\delta$ remains constant in the last value calculated $C$. This until a new event is detected. In this case, the initial conditions of the integral are reset and the input is connected again. In a multievent scenario (bottom of Fig. 3) i.e., if a second event is detected before the protocol has finished, the count and initial conditions are reset, and $k(t)$ starts again from $k_{\text {max }}$.

At the beginning of the protocol, a high value of $k(t)$ is proposed in order to obtain a fast transient response even if the error in frequency is high at this moment. The interval with constant $k(t)$ is used to facilitate the power sharing fastly. In the next interval, the ramp slope is low in order to do not introduce serious interactions between power sharing and frequency restoration. As $k(t)$ becomes smaller, the frequency error decreases until it is completely negligible. All these features will be validated experimentally in Section V.

\section{Event detection strategies}

The event detection strategy is important to guarantee a good performance of the proposed control in multiple scenarios. Notice that once the event is detected, the timedependent protocol works independently. However, considering the possibility of multi-event scenarios it is key to design a suitable event detection strategy.

One of the most common detection strategies is the so called send-on-delta (SOD) in which the event is triggered if a predefined signal deviates more than a threshold [27]. For this application (i.e., islanded microgrids with multiple DGs) the sampling should be performed over the local active power or the frequency. An advantage of event detection based on active power is that the changes on this variable are more appreciable, then easier to identify. However, event detection based on frequency is convenient considering that this is the only totally global variable in the microgrid.

For the purpose of this paper, two thresholds are used for the event detection: one based on the percentage of active power change and the other on frequency. In fact, the event is detected when at least one of the thresholds is exceeded. It is important to clarify that other possible event detection strategies are also possible for the proposed control. A deeper study of the impact of different event detection strategies on multi-event islanded microgrids is out of the scope of this paper and is left for future works.

\section{DESIGN CONSIDERATIONS}

The proposed switched control scheme and the timedependent protocol have four design parameters that determine the response of the secondary layer: $k_{i}$ which is related to the control scheme and $k_{\max }, \Delta_{c t}$, and $\Delta_{\text {ramp }}$ which are related to the time-dependent protocol. In the next subsections, design considerations to determine the values of these parameters and a stability analysis are presented. These 
TABLE I

PARAMETERS OF THE LABORATORY PROTOTYPE

\begin{tabular}{ccc}
\hline \hline Parameter & Symbol & Quantity \\
\hline Reference angular frequency & $\omega_{0}$ & $2 \pi \cdot 60 \mathrm{rad} / \mathrm{s}$ \\
Reference amplitude (peak value) & $V_{0}$ & $110 \cdot \sqrt{2} \mathrm{~V}$ \\
Microgrid nominal power & $S$ & $5 \mathrm{kVA}$ \\
Output inductors & $L$ & $5 \mathrm{mH}$ \\
Output capacitors & $C$ & $2 \mu \mathrm{F}$ \\
Line impedance 1 & $Z_{1}$ & $0.5+1.13 \mathrm{j} \Omega$ \\
Line impedance 2 & $Z_{2}$ & $0.5+0.38 \mathrm{j} \Omega$ \\
Line impedance 3 & $Z_{3}$ & $1.13+0.23 \mathrm{j} \Omega$ \\
Line impedance 4 & $Z_{4}$ & $0.3 \mathrm{j} \Omega$ \\
Main load & $L_{\operatorname{main}}$ & $1.5 \mathrm{~kW}$ \\
Local load 1 & $L_{1}$ & $0.5 \mathrm{~kW}$ \\
Virtual impedance & $Z_{v}$ & $3.393 \mathrm{j} \Omega$ \\
Droop-control gain & $m$ & $0.001 \mathrm{rad} /(\mathrm{W} \cdot \mathrm{s})$ \\
Primary layer cut-off frequency & $\omega_{c}$ & $2 \pi \mathrm{rad} / \mathrm{s}$ \\
Secondary layer parameter & $k_{i}$ & $90 \mathrm{rad} / \mathrm{s}$ \\
Maximum $k$ gain & $k_{\max }$ & 0.3 \\
Time interval for constant $k$ & $\Delta_{c t}$ & $5 \mathrm{~s}$ \\
Time interval for ramp $k$ & $\Delta_{\text {ramp }}$ & $5 \mathrm{~s}$ \\
\hline \hline
\end{tabular}

control design guidelines constitute the second contribution of this work.

\section{A. Closed-loop system modeling}

Fig. 4 illustrates the equivalent circuit of an electronicallycoupled DG system. Note that $u$ is the output voltage of the DG with $u=V \sin (\varnothing)$, where the phase is $\varnothing=\omega t+\varphi$, and $u_{b}$ is the main bus voltage with $u_{\mathrm{b}}=V_{\mathrm{b}} \sin \left(\emptyset_{\mathrm{b}}\right)$, where the phase is $\emptyset_{\mathrm{b}}=\omega t+0^{\circ} . Z_{e q}$ is the coupling impedance between the DG and the main bus, which can be calculated considering the output impedance of the DG to the microgrid and the virtual output impedance $Z_{v}$ [28]-[31]. If the coupling impedance is mainly inductive and the angle $\varphi$ approaches to zero (assuming $\sin (\varphi) \approx \varphi$ ), the output active power of the inverter can be calculated as follows

$$
p \approx \frac{3 V V_{\mathrm{b}}}{2 X_{e q}} \varphi=\frac{3 V V_{\mathrm{b}}}{2 X_{e q}} \frac{\omega}{s}=G_{\omega \mathrm{p}} \omega
$$

where $X_{e q}$ is the module of $Z_{e q}$ and $G_{\omega \mathrm{p}}$ is the system plant.

For design purposes, the closed-loop system equation for $k(t)>0$, can be derived by inserting (10) in (5) and (9), resulting in (11). This transfer function is used below to design the control parameters.

\section{B. Design of the control parameters}

A step-by-step procedure for the design of the control parameters is given below. This procedure uses as a starting point the values of the laboratory microgrid components and primary control parameters listed in Table I.

Firstly, the parameter $k_{\max }$ is designed. For this, it is assumed that the time of the protocol in which $k(t)=k_{\max }$

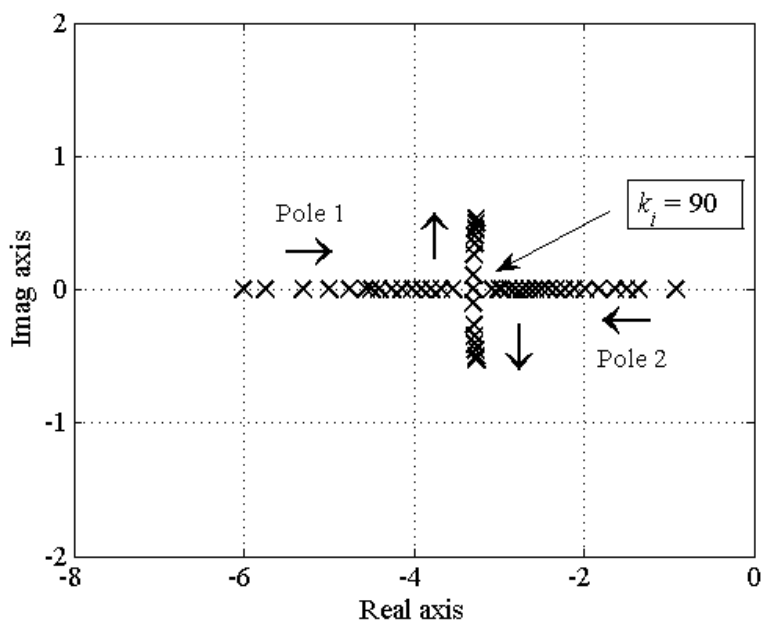

Fig. 5. Dominant closed-loop poles plot for $5 \leq k_{i} \leq 150, k=0.3$

(i.e., $\Delta_{c t}$ ) is large enough that frequency reaches an steadystate value. Then, from (7) a maximum frequency error is expressed as follows

$$
e_{0_{\max }}=\frac{k_{\max } m P_{\max }}{1+k_{\max }}
$$

where $P_{\max }$ is the maximum power delivered by a single DG. Assuming a desired maximum error of approximately $0.1 \%$, the value of $k_{\max }$ can be 0.3 . The stability related to the change of $k(t)$ is analyzed in the next subsection.

Secondly, the integral gain $k_{i}$ is designed taking in mind a desired location for the closed-loop poles. Using (11) and the usual approximation $V_{0}=V_{\mathrm{b}}=V$, a plot of the dominant closed-loop poles as a function of $k_{i}$ is represented in Fig. 5. The arrows indicate the increase of $k_{i}$. A good closed-loop dynamics is expected selecting $k_{i}=90 \mathrm{rad} / \mathrm{s}$, once the two poles form a double real pole. This will produce a fast dynamic with no overshoots.

Finally, the parameters $\Delta_{c t}$ and $\Delta_{\text {ramp }}$ are selected taking into consideration the design trade-off between transient response and control layer interactions. On one hand, the parameters should be designed with a high value in order to avoid interactions between power sharing and frequency restoration mechanisms. On the other hand, they should be selected with a low value in order to get a fast transient response. A good solution is achieved by selecting the total time of the protocol larger than the primary layer response. From Table I, note that the period associated to the primary layer cut-off frequency is $T_{c}=1 \mathrm{~s}$. Then, the total time is chosen a decade above resulting in $\Delta_{c t}+\Delta_{\text {ramp }}=10 \mathrm{~s}$. Furthermore, to achieve a good trade-off between the design characteristics previously discussed, $\Delta_{c t}$ and $\Delta_{\text {ramp }}$ are selected with the same value, then $\Delta_{c t}=5 \mathrm{~s}$ and $\Delta_{r a m p}=5 \mathrm{~s}$. This solution is excellent in case of multiple events, in which transient response is a critical performance index.

$$
\frac{\omega}{\omega_{0}}=\frac{s^{3}+s^{2}\left(\omega_{c}+k k_{i}+k_{i}\right)+s \omega_{c}\left(k k_{i}+k_{i}\right)}{s^{3}+s^{2}\left(\omega_{c}+k k_{i}+k_{i}\right)+s\left(\omega_{c}\left(k k_{i}+k_{i}\right)+m \frac{3 \omega_{c} V V_{\mathrm{b}}}{2 X_{e q}}\right)+m \frac{3 \omega_{c} V V_{\mathrm{b}} k k_{i}}{2 X_{e q}}}
$$




\section{Stability analysis}

The change in the variable $k(t)$ is a critical point for system stability. Proving it requires advanced stability methods because it does not suffice to check stability for each particular value of $k(t)$ separately (i.e., proof that the closed loop poles for each $k$-dependent system are in the left-plane). It is known that the combination of stable systems can lead to instability [32].

Hence, the approach used is to find a common Lyapunov function for the desired range of values for $k(t)$. To do so, closed-loop dynamics is transformed into matrix form. The plant and the control laws are expressed as follows

$$
\dot{x}=A x
$$

giving as closed-loop state matrix (detailed derivation is presented in Appendix A)

$$
\boldsymbol{A}=\left[\begin{array}{ccc}
-\omega_{c} & 0 & \frac{3}{2} \frac{V V_{b}}{X_{e q}} \omega_{c} \\
m k_{i} \operatorname{sgn}(k) & -k_{i}(1+k) \operatorname{sgn}(k) & 0 \\
-m & 1 & 0
\end{array}\right]
$$

and the state vector $\boldsymbol{x}$ defined as $\boldsymbol{x}=[P, \delta, \varphi]^{\mathrm{T}}$. Note that $\boldsymbol{A}$ depends on $k$.

According to Lyapunov stability [33], for parametervarying linear systems, a common Lyapunov function

$$
V(x)=\boldsymbol{x}^{T} \boldsymbol{M} \boldsymbol{x}
$$

for the given range of $k$ values should be found, with $\boldsymbol{M}$ satisfying

$$
\boldsymbol{A}^{T} \boldsymbol{M}+\boldsymbol{M A} \prec 0
$$

for some values that make $\boldsymbol{M}$ a positive defined matrix. Knowing that the design range is $0 \leq k \leq 0.3$, the Lyapunov function characterized by the values presented in Appendix B ensures stability (in fact, it guarantees stability for the range $0 \leq k \leq 1)$.

\section{EXPERIMENTAL RESULTS}

This Section presents selected experimental results that validate the expected features of both the proposed control scheme and time-dependent protocol.

A low power three-phase laboratory microgrid has been built following the scheme of Fig. 6. It consists of a dc source (AMREL SPS-800-12) which emulates the power generation of the DGs, distributed lines implemented by $R-L$ impedances and resistive loads for the power consumption. Each generator is connected to the microgrid bus through a MTLCBI0060F12IXHF GUASCH three-phase IGBT full-bridge power inverter and an $L-C$ output filter. The controller of each inverter was implemented on a F28M36 digital signal processor (DSP) from Texas Instruments. The experimental setup is shown in Fig. 7.

The experimental tests reproduce the black start of the microgrid. DGs and loads are connected sequentially, except

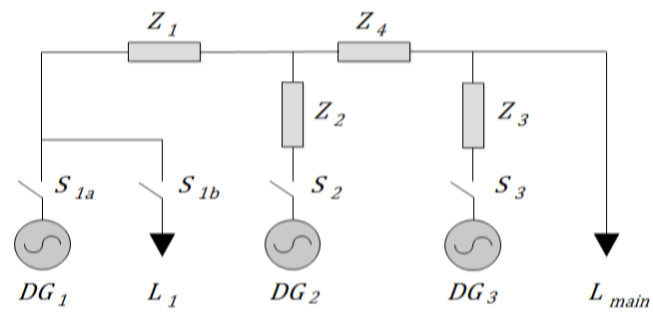

Fig. 6. Single-phase diagram of the laboratory microgrid

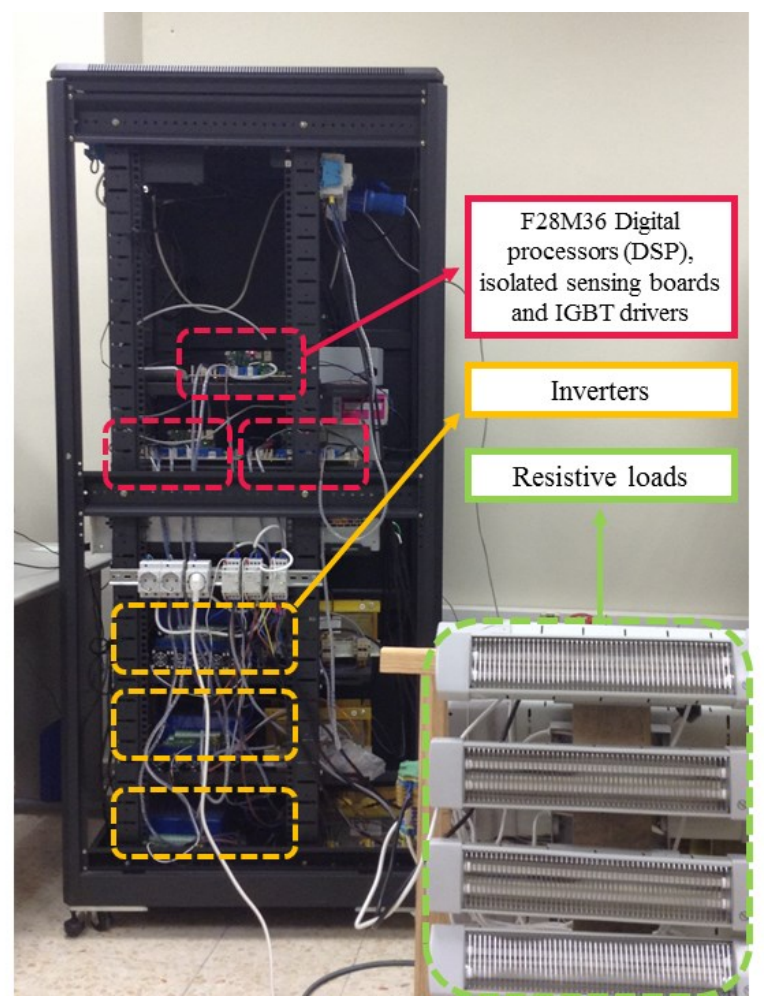

Fig. 7. Experimental setup

the main load $L_{\text {main }}$ that remains connected all the time. All the DGs work with converters programmed as grid-forming (power-controlled voltage sources) except in the last test, as is explained below. A phase-locked loop based voltage synchronization procedure is performed in all the controllers of the DGs, facilitating the soft start of these units [26].

The experiment begins with the soft start of $\mathrm{DG}_{1}$ connected through the switch $S_{I a}$ supplying the main load $L_{\text {main }}$. At $\mathrm{t}=20 \mathrm{~s}$ switch $S_{2}$ closes connecting $\mathrm{DG}_{2}$ to the microgrid, then the load sharing of $L_{\text {main }}$ begins. At $\mathrm{t}=40 \mathrm{~s}$ switch $S_{l b}$ closes connecting local load $L_{l}$ and changing the total load of the microgrid. Finally, at $\mathrm{t}=60 \mathrm{~s}$, switch $S_{3}$ closes connecting $\mathrm{DG}_{3}$. In subsection $\mathrm{F}$ an experimental test with a dynamicallychanging load and different droop gains is presented.

\section{A. Test using only primary control layer}

First, a test using only primary control layer was performed. The same value of $m$ was implemented for all the converters (see Table I) considering DGs with equal nominal powers. However, it is possible to achieve a desired proportional power sharing designing different droop gains for each DG without affecting the dynamic response of the control layers, as is presented in Section V.F. 


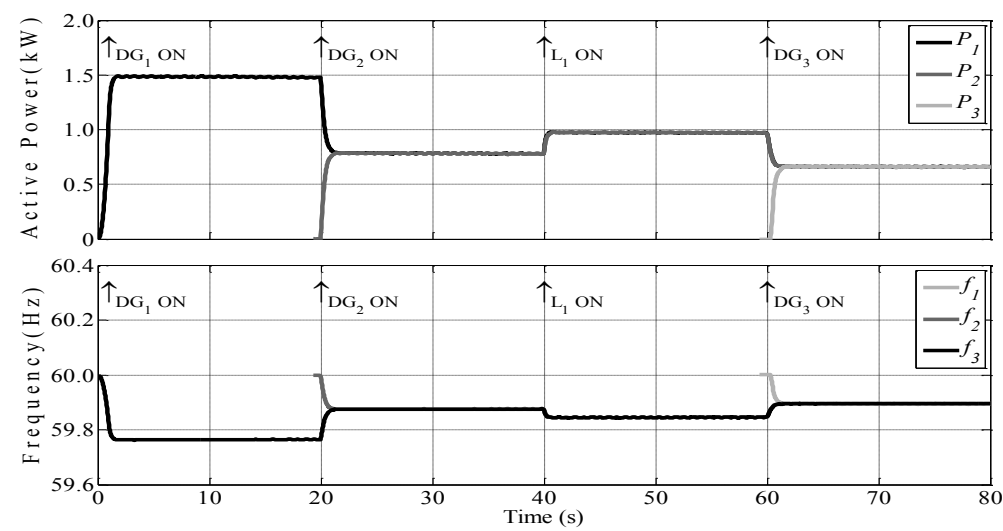

Fig. 8. Active power and frequency using only primary control layer.

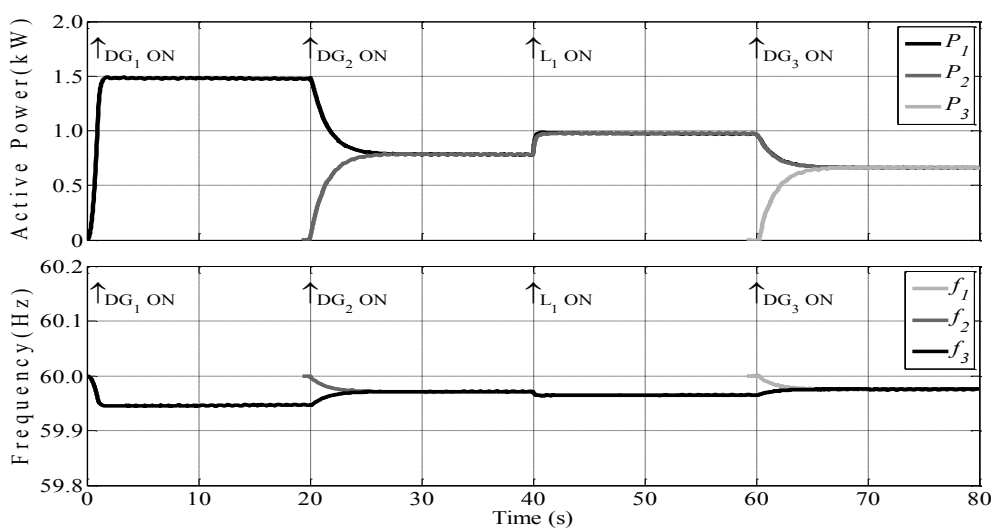

(a)

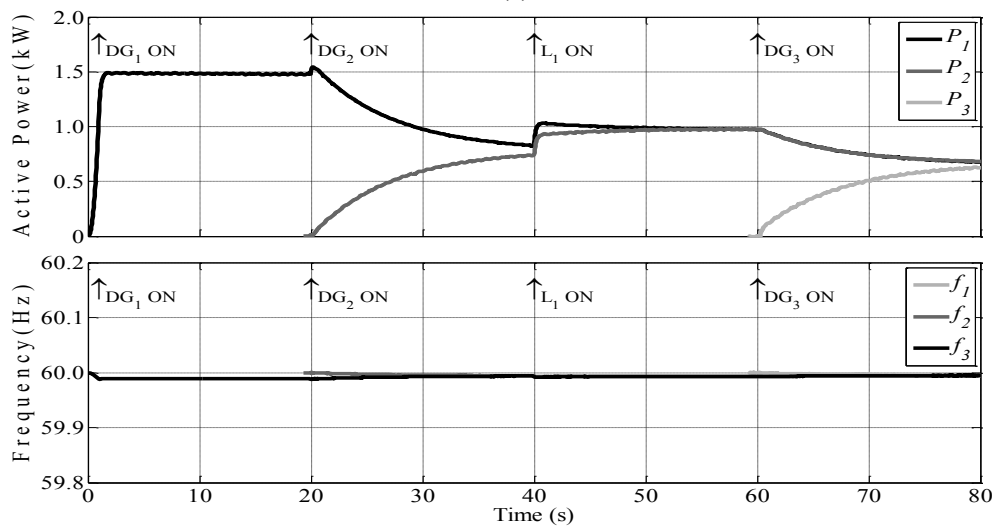

(b)

Fig. 9. Active power and frequency using invariant low pass filters, with (a) $k_{0}=0.3$ and (b) $k_{0}=0.05$.

In Fig. 8 active power and frequency are shown. Primary layer performs a fast an accurate power sharing, however, the droop characteristic produces a considerable deviation in frequency. As will be shown in the following tests, secondary control layer is introduced to reduce this frequency error.

\section{B. Tests using invariant low-pass filters}

Tests using secondary control layer based on invariant lowpass filters (6) were performed to verify the trade-off discussed in previous sections. In Fig. 8 active powers and frequencies for $k_{i}=90 \mathrm{rad} / \mathrm{s}$ with $k_{0}=0.3$ and $k_{0}=0.05$ are shown. In Fig. 9(a) it can be noted that with the higher value of $k_{0}$ secondary layer performs a fast and accurate power sharing in $5 \mathrm{~s}$ with a more appreciable frequency error in steady-state. On the other hand, in Fig. 9(b) with the lower value of $k_{0}$ the dynamic has a noticeably slower power sharing response, with a frequency error highly reduced.

These tests can be used as a benchmark to appreciate the advantages of the control proposed over the limited proposals found in the literature, in particular over the trade-off between transient response and accuracy. In the next subsection, the proposed control with no communications is tested showing simultaneously fast transient response and an accurate steadystate frequency. 


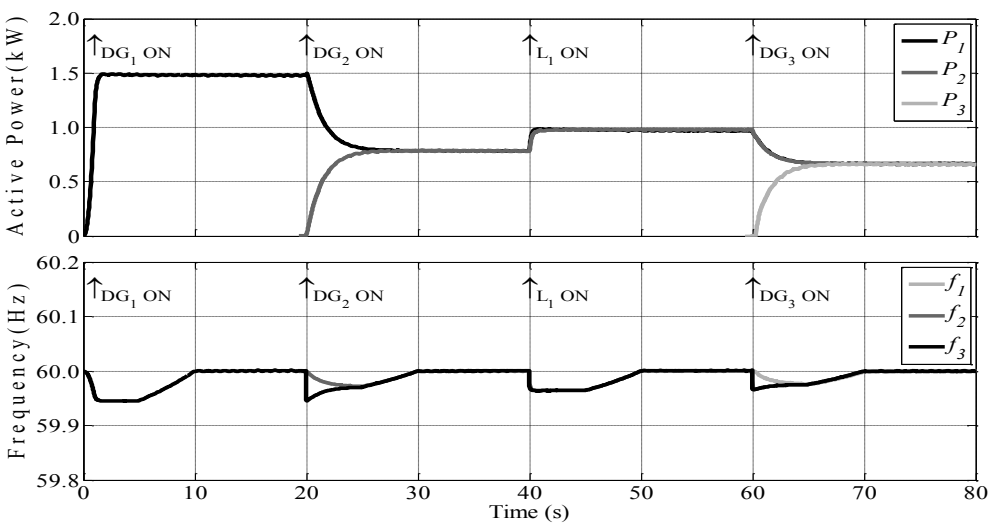

Fig. 10. Active power and frequency of a test using the proposed control.

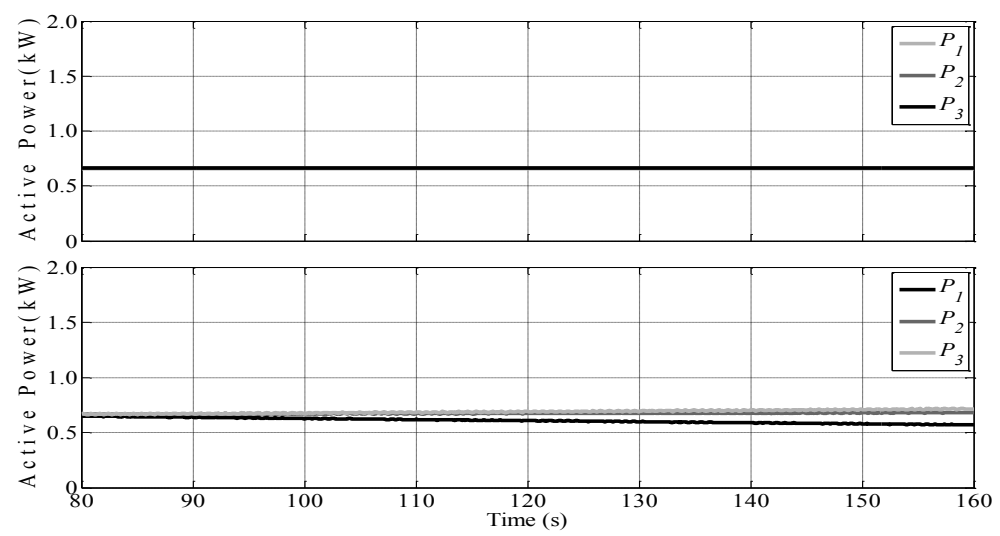

Fig. 11. Active powers with switched control (top) and without switched control (bottom).
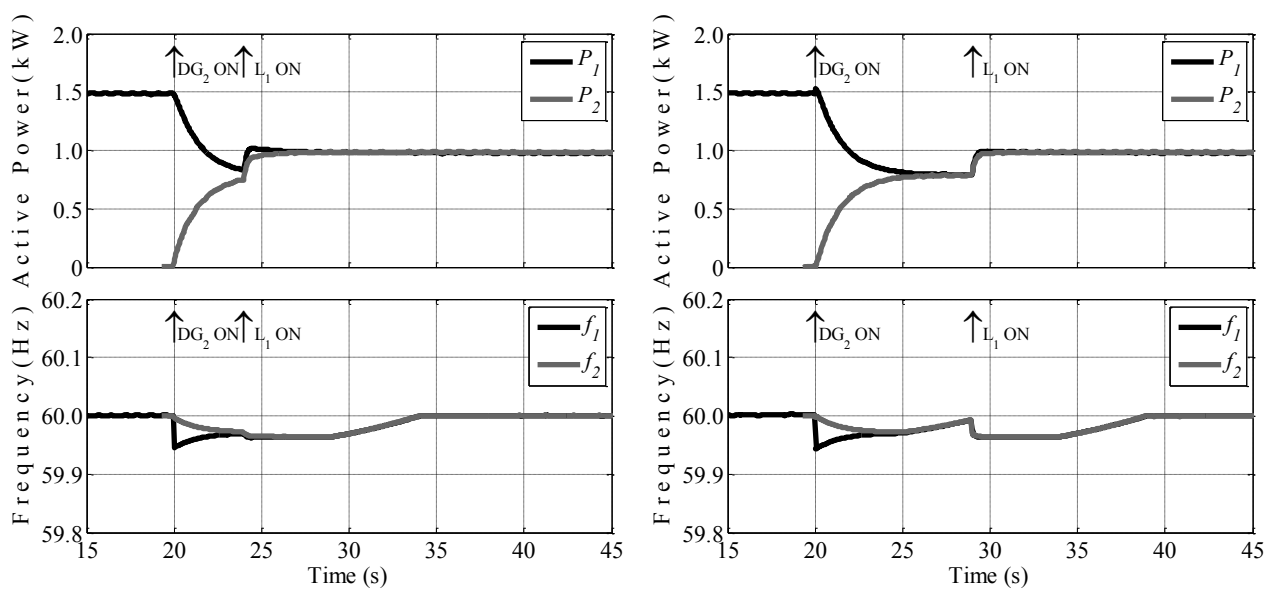

Fig. 12. Active powers and frequencies for a multi-event situation with event detection in $t=20 \mathrm{~s}$ and in $t=24 \mathrm{~s}$ (left) or $t=29 \mathrm{~s}$ (right).

\section{Tests using the proposed control}

Fig. 10 shows the active power and frequency using the proposed control. During the initial $k$-constant interval $\left(\Delta_{c t}\right)$ an accurate power sharing is done in $5 \mathrm{~s}$, however an appreciable error in the frequency recovery can be noted. In the next interval, the linear variation of $k(t)$ from $k_{\max }$ to 0 leads to the compensation of the frequency error, showing a good dynamic response.

It is worth to mention that the transient response is faster during a load change (40s) than during a DG connection (20s and 60s). The reason is the difference on the frequencies at the beginning of the protocol: in a load change, all the DGs work at the same frequency, contrary to the start of a DG due to the effect of the load consumption.

A sensitivity analysis was carried out for the parameters $k_{i}$ and $k_{\max }$. Deviations of $\pm 2.5 \%, \pm 5 \%$ and $\pm 10 \%$ were considered separately for both parameters in each particular DG controller. It is worth mentioning that the frequency accurately reached the expected value in all the cases. In addition, the deviation in power sharing caused by changes in $k_{i}$ are completely negligible. However, for $k_{\max }$, the power sharing experienced deviations of $\pm 2.3 \%, \pm 4.2 \%$ and $\pm 9.1 \%$. 


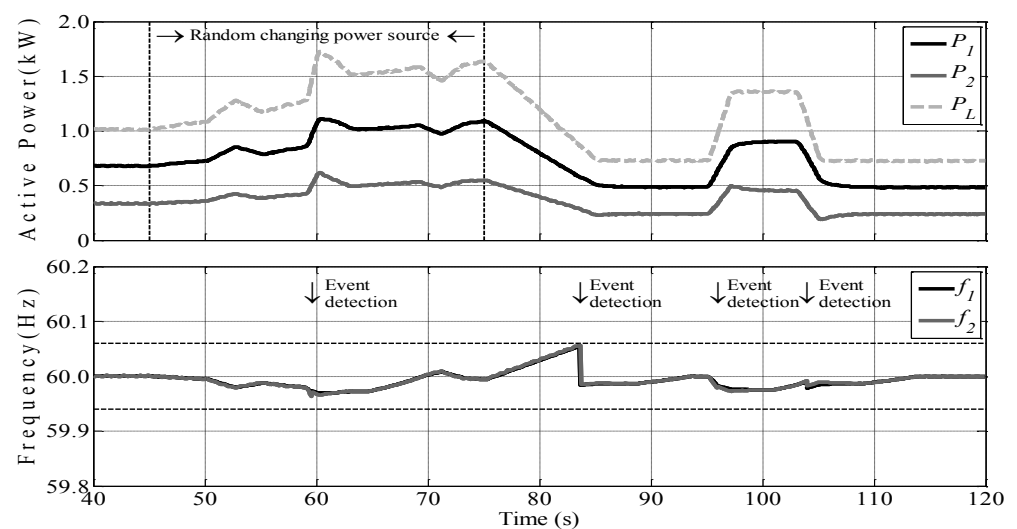

Fig. 13. Active power and frequency of a test with a dynamically-changing load and different droop gains.

Therefore, in order to get a good power sharing, the parameter $k_{\max }$ must present no mismatches in the practical implementation of the controllers. This is really easy to achieve in practice because of digital technology used for the control implementation.

\section{Tests without the switched control}

The next tests were done to analyze the relevance of the switched characteristic in the proposed control. As was previously discussed, if the switched characteristic is not implemented, the control scheme works as an integral control and suffers the hunting phenomenon which leads to unstable responses. In Fig. 11 the active power for tests with switched control (top) and without switched control (bottom) are presented.

Notice that the time interval shown is $80 \mathrm{~s} \leq t \leq 160 \mathrm{~s}$ (when there are no load changes) because the difference between both tests only can be appreciated several seconds later the time-dependent protocol has finished. Thus, is possible to highlight the relevance of the switched characteristic in the proposed control to avoid the drawbacks of integral controllers.

\section{E. Tests with multi-event scenario}

The next test (Fig. 12) shows the behavior of the system in a two-event scenario. To the left, active power and frequency are shown for a multi-event situation with the first event detected $\left(\mathrm{DG}_{2}\right.$ startup) at $\mathrm{t}=20 \mathrm{~s}$ and a second event detected (load change) at $\mathrm{t}=24 \mathrm{~s}$. As the protocol restarts during the $k$ constant interval, when the power sharing transitory is not finished, the active powers are still different just when the second event is detected. However, a few seconds later the power sharing is completed showing good dynamic characteristics.

To the right, active power and frequency are shown for a multi-event situation with the first event detected $\left(\mathrm{DG}_{2}\right.$ startup) at $\mathrm{t}=20 \mathrm{~s}$ and a second event detected (load change) at $\mathrm{t}=29 \mathrm{~s}$. In this case, the second event detection is done $1 \mathrm{~s}$ before the end of the protocol. For this reason, the power sharing was already done. In both cases, the proposed protocol shows a fast and accurate response in active power and frequency.

\section{F. Test with a dynamically-changing load and different droop gains}

The last test was done to analyze the proposed control in the presence of a dynamically-changing load and different droop gains. The changing load is simulated programming the controller of $\mathrm{DG}_{3}$ as a grid-feeding current source and keeping $L_{\text {main }}$ and $L_{1}$ connected during all the test. Thus, the equivalent load $P_{L}$ corresponds to $P_{L}=L_{\text {main }}+L_{1}-P_{3}$. Controllers of $\mathrm{DG}_{1}$ and $\mathrm{DG}_{2}$ work programmed as gridforming voltage sources. For this reason, the changes on $P_{L}$ produce variations in the injected powers $P_{1}$ and $P_{2}$. For $\mathrm{DG}_{1}$ and $\mathrm{DG}_{2}$ different values of droops gains have been programmed (i.e., $m_{1}=0.00075$ and $m_{2}=0.0015$ ), with an inherent effect over the proportionality of the power sharing. Active power and frequency are shown in Fig. 13.

During the initial $40 \mathrm{~s}, \mathrm{DG}_{1}$ and $\mathrm{DG}_{2}$ were soft started while $P_{L}$ has a constant power of $1000 \mathrm{~W}$. This period of time was omitted considering that does not present relevant information compared with the analyzed in previous tests. From $\mathrm{t}=45 \mathrm{~s}$ to $\mathrm{t}=75 \mathrm{~s}, P_{L}$ behaves as a random changing load. At $\mathrm{t}=59.6 \mathrm{~s}$ an event is detected by the excess of the power threshold and the protocol is started. It is worth mentioning that the threshold is based on the percentage of power change, for this reason despite the difference in the powers delivered, the event is correctly detected.

Notice that just when the protocol ends, the frequency is restored (i.e., is equal to $60 \mathrm{~Hz}$ ). However, as the random changes in the load remain, the frequency is affected by these fluctuations. This operation coincides with that expected in other secondary control schemes, which eliminate the frequency deviation only in steady state.

At $\mathrm{t}=75 \mathrm{~s}, P_{L}$ varies linearly from $1700 \mathrm{~W}$ to $700 \mathrm{~W}$ in an interval of $10 \mathrm{~s}$. The change in the power generated by $\mathrm{DG}_{1}$ and $\mathrm{DG}_{2}$ produces a linear variation in frequency which exceeds the threshold of event detection at $\mathrm{t}=83.7 \mathrm{~s}$, then starting the protocol. Finally, at $\mathrm{t}=95 \mathrm{~s}$ and $\mathrm{t}=103 \mathrm{~s} P_{L}$ increases and decreases between $700 \mathrm{~W}$ and $1400 \mathrm{~W}$, both in intervals of $2 \mathrm{~s}$. In these cases, events are detected by the excess of the power change threshold in times that produce a multi-event scenario.

This last test clearly shows that even in the presence of a dynamically-changing load and DGs with different droop gains, the proposed control guarantees a suitable working 
frequency in both steady state and transient scenarios. In steady-state, an accurate frequency recovery is done, as shown in the different tests presented. In transients, the frequency remains in a working margin although the power changes, as a consequence of the frequency threshold implemented. For this reason, a good design of the event detection strategy results key to achieve a good performance of the control proposed, as discussed in Section III.C.

\section{CONCLUSIONS}

This paper proposes a secondary control with no communications for islanded microgrids. It is based on a switched control scheme that uses a time-dependent protocol to change between two control configurations. With this structure, an excellent performance in terms of flexibility, reliability, transient response and frequency restoration in steady-state is achieved simultaneously. The design trade-off found in previous controllers between transient response and frequency restoration is broken with the proposed control solution, thus improving clearly the performance of the stateof-the-art control schemes with no communications. In addition, design considerations of the proposed control are presented. Experimental results are reported, confirming the validity of the predicted characteristics.

Future work will be focused on extending the main ideas of this proposal to the reactive power-voltage control loop to achieve a robust voltage control with no communication. Also, study in depth the performance of different event detection strategies on multi-event scenarios of microgrids is an open topic of research.

\section{APPENDIX}

\section{A. Derivation of closed-loop state matrix}

The active power is derived by inserting the time domain representation of the plant given in (10) in (3):

$$
\frac{d P}{d t}=\frac{3}{2} \frac{V V_{b}}{X_{e q}} \omega_{c} \varphi-\omega_{c} P .
$$

The secondary control is deduced by inserting (5) in (8):

$$
\frac{d \delta}{d t}=m k_{i} \operatorname{sgn}(k) P-k_{i}(\operatorname{sgn}(k)+k) \delta .
$$

For convenience, the frequency is rewritten as

$$
\omega=\frac{d \emptyset}{d t}=\frac{d\left(\omega_{o} t+\varphi\right)}{d t}=\omega_{o}+\frac{d \varphi}{d t} .
$$

Then, inserting (5) in (A3), the initial phase $\varphi$ is expressed as

$$
\frac{d \varphi}{d t}=-m P+\delta
$$

The matrix $\boldsymbol{A}$ can be easily written as (14) using (A1), (A2), and (A4).

\section{B. Values of matrix $\boldsymbol{M}$}

$$
\boldsymbol{M}=\left[\begin{array}{ccc}
0.0000558 & -0.0243840 & -0.2364176 \\
-0.0243840 & 36.46885 & 23.1569566 \\
-0.2364176 & 23.1569566 & 11873.5845121
\end{array}\right] \cdot 10^{-6}
$$

\section{REFERENCES}

[1] N. Hatziargyriou, H. Asano, R. Iravani, and C. Marnay, "Microgrids," IEEE Power Energy Mag., vol. 5, no. 4, pp. 7894, 2007.

[2] K. Moslehi and R. Kumar, "A reliability perspective of the smart grid," IEEE Trans. Smart Grid, vol. 1, no. 1, pp. 57-64, 2010.

[3] S. Parhizi, H. Lotfi, A. Khodaei, and S. Bahramirad, "State of the art in research on microgrids: a review," IEEE Access, vol. 3, no. 1, pp. 890-925, 2015

[4] X. Sun, B. Liu, Y. Cai, H. Zhang, Y. Zhu, and B. Wang, "Frequency-based power management for photovoltaic/battery/fuel cell-electrolyser stand-alone microgrid," IET Power Electron., vol. 9, no. 13, pp. 26022610, Oct. 2016.

[5] J. Vasquez, J. M. Guerrero, J. Miret, M. Castilla, and L. Garcia de Vicuña, "Hierarchical control of intelligent microgrids," IEEE Ind. Electron. Mag., vol. 4, no. 4, pp. 23-29, Dec. 2010.

[6] G. Chen, F. L. Lewis, E. N. Feng, and Y. Song, "Distributed optimal active power control of multiple generation systems," IEEE Trans. Ind. Electron., vol. 62, no. 11, pp. 7079-7090, Nov. 2015.

[7] N. Lakshminarasamma, S. Augustine, and M. K. Mishra, "Control of photovoltaic-based low-voltage dc microgrid system for power sharing with modified droop algorithm," IET Power Electron., vol. 9, no. 6, pp. 1132-1143, May 2016.

[8] J. Yu, C. Dou, and X. Li, "MAS-Based energy management strategies for a hybrid energy generation system," IEEE Trans. Ind. Electron., vol. 63, no. 6, pp. 3756-3764, Jun. 2016.

[9] J. A. P. Lopes, C. L. Moreira, and A. G. Madureira, "Defining control strategies for microgrids islanded operation," IEEE Trans. Power Syst., vol. 21, no. 2, pp. 916-924, May 2006.

[10] D. Wu, F. Tang, T. Dragicevic, J. C. Vasquez, and J. M. Guerrero, "Autonomous active power control for islanded AC microgrids with photovoltaic generation and energy storage system," IEEE Trans. Energy Convers., vol. 29, no. 4, pp. 882-892, Dec. 2014

[11] Q. Shafiee, J. M. Guerrero, and J. C. Vasquez, "Distributed secondary control for islanded microgrids - A novel approach," IEEE Trans. Power Electron., vol. 29, no. 2, pp. 1018-1031, Feb. 2014.

[12] Q. Shafiee, C. Stefanovic, T. Dragicevic, P. Popovski, J. C. Vasquez, and J. M. Guerrero, "Robust networked control scheme for distributed secondary control of islanded microgrids," IEEE Trans. Ind. Electron., vol. 61, no. 10, pp. 5363-5374, Oct. 2014.

[13] L. Meng, X. Zhao, F. Tang, M. Savaghebi, T. Dragicevic, J. C. Vasquez, and J. M. Guerrero, "Distributed voltage unbalance compensation in islanded microgrids by using a dynamic consensus algorithm," IEEE Trans. Power Electron., vol. 31, no. 1, pp. 827-838, Jan. 2016.

[14] F. Guo, C. Wen, J. Mao, and Y.-D. Song, "Distributed secondary voltage and frequency restoration control of droopcontrolled inverter-based microgrids," IEEE Trans. Ind. Electron., vol. 62, no. 7, pp. 4355-4364, Jul. 2015.

[15] L.-Y. Lu and C.-C. Chu, "Consensus-based droop control synthesis for multiple DICs in isolated micro-grids," IEEE Trans. Power Syst., vol. 30, no. 5, pp. 2243-2256, Sep. 2015.

[16] L.-Y. Lu and C.-C. Chu, "Consensus-based secondary frequency and voltage droop control of virtual synchronous generators for isolated AC micro-grids," IEEE J. Emerg. Sel. Top. Circuits Syst., vol. 5, no. 3, pp. 443-455, Sep. 2015. 
[17] J. W. Simpson-Porco, Q. Shafiee, F. Dorfler, J. C. Vasquez, J. M. Guerrero, and F. Bullo, "Secondary frequency and voltage control of islanded microgrids via distributed averaging," IEEE Trans. Ind. Electron., vol. 62, no. 11, pp. 7025-7038, Nov. 2015.

[18] C. Ahumada, R. Cardenas, D. Saez, and J. M. Guerrero, "Secondary control strategies for frequency restoration in islanded microgrids with consideration of communication delays," IEEE Trans. Smart Grid, vol. 7, no. 3, pp. 14301441, May 2016.

[19] S. Liu, X. Wang, and P. X. Liu, "Impact of communication delays on secondary frequency control in an islanded microgrid," IEEE Trans. Ind. Electron., vol. 62, no. 4, pp. 2021-2031, Apr. 2015.

[20] V. Mariani, F. Vasca, J. C. Vasquez, and J. M. Guerrero, "Model order reductions for stability analysis of islanded microgrids with droop control," IEEE Trans. Ind. Electron., vol. 62, no. 7, pp. 4344-4354, Jul. 2015.

[21] E. A. Coelho, D. Wu, J. M. Guerrero, J. C. Vasquez, T. Dragicevic, C. Stefanovic, and P. Popovski, "Small-signal analysis of the microgrid secondary control considering a communication time delay," IEEE Trans. Ind. Electron., vol. 63, no. 10, pp. 6257-5269, 2016.

[22] H. Shi, Z. Fang, H. Yi, F. Wang, D. Zhang, and Z. Geng, "A novel real-time voltage and frequency compensation strategy for photovoltaic-based microgrid," IEEE Trans. Ind. Electron., vol. 62, no. 6, pp. 1-1, 2014.

[23] H. Xin, L. Zhang, Z. Wang, D. Gan, and K. P. Wong, "Control of island AC microgrids using a fully distributed approach," IEEE Trans. Smart Grid, vol. 6, no. 2, pp. 943-945, Mar. 2015.

[24] H. Xin, R. Zhao, L. Zhang, Z. Wang, K. P. Wong, and W. Wei, "A decentralized hierarchical control structure and selfoptimizing control strategy for F-P type DGs in islanded microgrids," IEEE Trans. Smart Grid, vol. 7, no. 1, pp. 3-5, Jan. 2016.

[25] M. Hua, H. Hu, Y. Xing, and J. M. Guerrero, "Multilayer control for inverters in parallel operation without intercommunications," IEEE Trans. Power Electron., vol. 27, no. 8, pp. 3651-3663, 2012.

[26] J. M. Guerrero, J. C. Vasquez, J. Matas, L. García de Vicuña, and M. Castilla, "Hierarchical control of droop-controlled AC and DC microgrids-A general approach toward standardization," IEEE Trans. Ind. Electron., vol. 58, no. 1, pp. 158-172, Jan. 2011.

[27] B. A. Moser and T. Natschläger, "On stability of distance measures for event sequences induced by level-crossing sampling," IEEE Trans. Signal Process., vol. 62, no. 8, pp. 1987-1999, 2014.

[28] J. M. Guerrero, L. Garcia de Vicuña, J. Matas, M. Castilla, and J. Miret, "Output impedance design of parallel-connected UPS inverters with wireless load-sharing control," IEEE Trans. Ind. Electron., vol. 52, no. 4, pp. 1126-1135, Aug. 2005.

[29] J. Matas, M. Castilla, L. García de Vicuña, J. Miret, and J. C. Vasquez, "Virtual impedance loop for droop-controlled singlephase parallel inverters using a second-order generalintegrator scheme," IEEE Trans. Power Electron., vol. 25, no. 12, pp. 2993-3002, Dec. 2010.

[30] H. Cai, X. He, J. Shi, H. Zhao, and P. Zhang, "Power decoupling strategy based on 'virtual negative resistor' for inverters in low-voltage microgrids," IET Power Electron., vol. 9, no. 5, pp. 1037-1044, Apr. 2016.

[31] X. Wang, Y. W. Li, F. Blaabjerg, and P. C. Loh, "Virtualimpedance-based control for voltage-source and currentsource converters," IEEE Trans. Power Electron., vol. 30, no. 12, pp. 7019-7037, Dec. 2015.

[32] D. Liberzon, Switching in Systems and Control. Boston, MA: Birkhäuser Boston, 2003.

[33] J.-J. E. Slotine and L. I. Weiping, Applied Nonlinear Control. Englewood Cliff, New Jerse, 1991.

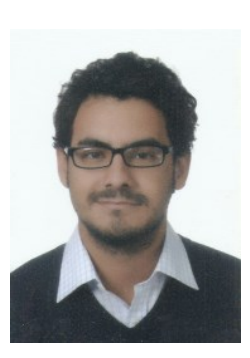

Juan M. Rey was born in Bucaramanga, Colombia in 1989. He received the B.S. in electrical engineering from Universidad Industrial de Santander, Bucaramanga, Colombia, in 2012. He is currently working toward the Ph.D. degree in the Department of Electronic Engineering, Technical University of Catalonia, Spain.

Since 2013, he has been with the Electrical, Electronic and Telecommunications Engineering School (E3T), Universidad Industrial de Santander, Bucaramanga Colombia, where he is currently an Assistant Professor. His research interest are power electronics and control for distributed generation and microgrids.

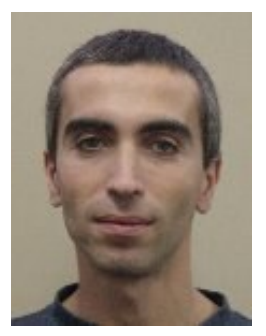

Pau Martí received the degree in computer science and the PhD degree in automatic control from the Technical University of Catalonia, Barcelona, Spain, in 1996 and 2002, respectively. From 2003 to 2004, he held a research fellow appointment in the Computer Science Department at the University of California at Santa Cruz, US. From 1996 to 2013, he has been an assistant professor in the Department of Automatic Control at the Technical University of Catalonia. Since 2013 he has been an Associate Professor in the Department of Automatic Control at the Technical University of Catalonia. His research interests include embedded and networked control systems, smart grids and microgrids.

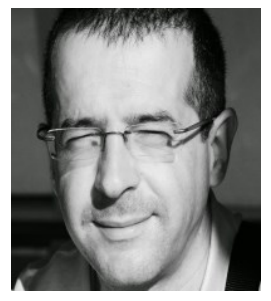

Manel Velasco received the degree in maritime engineering and the Ph.D. degree in automatic control from the Technical University of Catalonia, Barcelona, in 1999 and 2006, respectively. Since 2002, he has been an Assistant Professor in the Department of Automatic Control, Technical University of Catalonia. He has been involved in research on artificial intelligence from 1999 to 2002 and, since 2000 , on the impact of real-time systems on control systems. His research interests include artificial intelligence, real-time control systems, and collaborative control systems, especially on redundant controllers and multiple controllers with self-interacting systems.

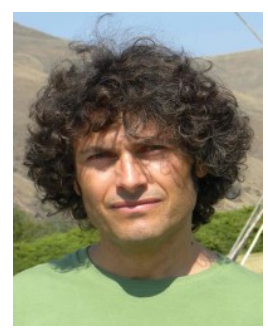

Jaume Miret (M'98) received the B.S. degree in telecommunications, M.S. degree in electronics, and Ph.D. degree in electronics from the Universitat Politecnica de Catalunya, Barcelona, Spain, in 1992, 1999, and 2005, respectively. From 1993 to 2011, he was an Assistant Professor in the Department of Electronic Engineering, Universitat Politecnica de Catalunya, Spain. Since 2011 he has been an Associate Professor in the Universitat Politecnica de Catalunya, where he teaches courses on digital design and circuit theory. His research interests include dc-to-ac converters, active power filters, and digital control.

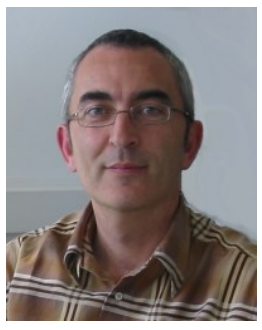

Miguel Castilla received the B.S., M.S., and Ph.D. degrees in telecommunication engineering from the Technical University of Catalonia, Barcelona, Spain, in 1988, 1995, and 1998, respectively. Since 2002, he has been an Associate Professor in the Department of Electronic Engineering, Technical University of Catalonia, where he teaches courses on analog circuits and power electronics. His research interests include the areas of power electronics, nonlinear control, and renewable energy systems. 